\title{
Diagnostic and Therapeutic Value of Cardiac Scan: First Experience of Utility and Prosfect of MPI in Bangladesh
}

\author{
A Azam ${ }^{1}$, AAS Majumder ${ }^{2}$, N Hossain², NAK Ahsan ${ }^{3}$, WA Jahan ${ }^{4}$, A Sabur ${ }^{3}$, S Ahmed $^{3}$, JH Khan $^{3}$, \\ AW Chowdhury ${ }^{2}$, AKM Mohibullah ${ }^{2}$ \\ ${ }^{1}$ Department of Nuclear Cardiology, ${ }^{2}$ Department of Cardiology, ${ }^{3}$ Department of Cardiac Surgery, \\ National Instiatute of Cardiovascular Diseases, ${ }^{4}$ Department of Radiology Bangabandhu Sheikh Mujib \\ Medical University, Dhaka
}

\begin{abstract}
Keywords:
Myocardial

viability,

Cardiac Scan

(Synoname: WI,

$M P-S P E C T$,

Thallium Scan).

$M P I$

Abstract:

Background: Cardiac Scan (MP-SPECT) is a widely utilized noninvasive imaging modality for diagnosis, prognosis, and risk stratification of coronary artery disease. In Bangladesh it is a recently introduced test and there is no study upon it. Considering this ground the study is perform on Bangladeshi patients.

Methods: 100 referral patients underwent MPI for evaluation of perfusion status of myocardium. The patients either of suspected IHD or diagnosed case of IHD were referred from different cardiology unit or surgery unit of NICVD. Technetium $99 m\left({ }^{99 " T c}\right)$ isotopes and tetrofosmin used in the same day stress and rest protocol.

Result: The commonest findings observed in this present analysis were the early age group patients mostly of female having DCM, but the later age group of patients are of both male and female having Angina Pectoris, OMI and ICM.

The referral patients by cardiologists or cardiac surgeons are mostly limited to the pre therapeutic evaluation rather than diagnostic indication.

The most common indication is the evaluation of myocardial viability and aim of subsequent treatment.

Conclusion: Analysis of perfusion status, decision of subsequent treatment either by medicine or $C A B G$, conform the usually described pattern in western literature.
\end{abstract}

(CVJ 2008; 1(1) : 53-58)

\section{Introduction:}

Over the past three decade myocardial perfusion single photon emission computed tomography (SPECT) has emerged as a robust tool for non invasive assessment of both myocardial perfusion and function. Since its inception in the 1970s, many advance have been made that have enhanced the diagnostic and prognostic strength of this modality, including the development of new Technetium 99m $\left({ }^{99} \mathrm{mTc}\right)$-based on isotopes, implementation of SPECT, Multidetector cameras, computerized quantification, attenuation correction, and ECG gating for assessment of LV function. These advances allow for very high diagnostic sensitivity and specificity. In addition there is wealth of data supporting the strength of this technique as a prognostic tool, not only in general population, but also in many important patient subgroups, such as women, patient with diabetes mellitus, in post revascularization patients, and as a preoperative assessment prior to noncardiac surgery.
In Bangladesh MPI is a newly introduced modem noninvasive test. In this study a total of 100 patients of both sex were evaluated by Myocardial Perfusion Imaging (MPI) in NICVD, Dhaka, Bangladesh. Full hemodynamic data, echo, ETT and in some cases CAG result were available. Even some patient has referred for MPI to see the post CABG or post PTCA status, so that prior diagnosis of coronary artery disease was established in those cases.

Technetium tetrofosmin were used as a perfusion reagent. Gated SPECT procedure was done under dual head gamma camera in same day protocol. Treadmill stress instead of pharmacological stress was used in conjunction with MPI. The scanning has done in 64 slices both for stress and rest. Processing and reconstruction of image has done both in rest and stress slices of heart in SA, VLA and HLA views. Interpretation of the result has done by visual comparison of sequential images of Stress with rest in numerical parallel of SA, VLA and HLA. 


\section{Materials and Methods:}

This was a prospective study done in the Nuclear Cardiology department of NICVD during the year 2003-2004 and May 2007-May 2008. Here I like to mention that due to technical problem of the machineries, MP-SPECT could not be performed during the period of 2005 -2007. In total 100 patients were included in the study in random but sequential manner.

Thursday of the week is the specific day of cardiac scan in NICVD, having a year long prior routine, published by authority of this institute at the beginning of the year. Here it is for information that Bangladesh Atomic Energy Commission (BAEC) is the provider of technetium, the main isotopes of cardiac scan and tetrofosmin, for NICVD. According to their suggestion the schedule of cardiac scan is to be made.

All the patients underwent cardiac perfusion were referred by cardiac surgeons or cardiologists. On reporting to the Nuclear Cardiology department the patient was advised to attend on schedule date with all available relevant documents for MPI. Thorough history was taken and meticulous physical examination was done in all cases. Laboratory investigations like ECG, ETT, X-ray, Echocardiophy, and CAG were done by referral cardiologist or cardiac surgeon. In addition CPC, Lipid Profile, Serum Uric Acid level, BUN, Urea, Creatinine also had had with patients.

On attending in $\mathrm{f}$ Nuclear Cardiology department, every thing related to scan were explained to patient. Then he was allowed to stress test according to standard international protocol and programme. At peak of exercise or achievement of THR, 10 mcuri technitium tetrofosmin injected and continue the exercise for more 60-90 seconds. Then scanning was taken in 64 slices by Gama Camera, conventionally after 30 minutes of 1 st injection. For rest scan the $2^{\text {nd }}$ injection of 20 mcuri technitium tetrofosmin were given after 10-15 minutes of rest and scanning in 64 slices by Gama Camera, after 30 minutes has completed.

Finally processing and reconstruction of MPI result in SA, VLA and HLA and comparing same section slices between rest and stress whether reversible or not in slandered method of interpretation. Similarly comparison of same slices in systole and diastole has done to see the wall thickness, wall motion, and chamber size as well as ejection fraction. Depending on the findings final reporting has made and supplied to the consultant.

\section{Results:}

1). Distribution of age of referral patients for MPI:

Age ranged from 21 to 80 years.

(i). 21 to 35 years $=10(10 \%)$.

(ii). 35 to 45 years $=27(27 \%)$.

(iii). $45-60$ years $=48$ (48\%) (iv). Above 60 years $=15$ (15\%).

(iv). Above 60 yrs $=15(15 \%)$.

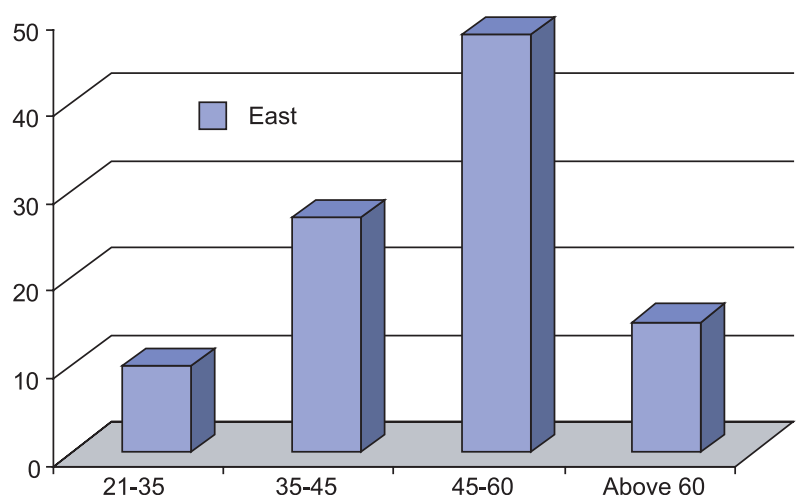

Fig.-1: Age distribution of referral patients for MPI

2). Sex distribution of referral patients for MPI:

Female 17; Male 83.

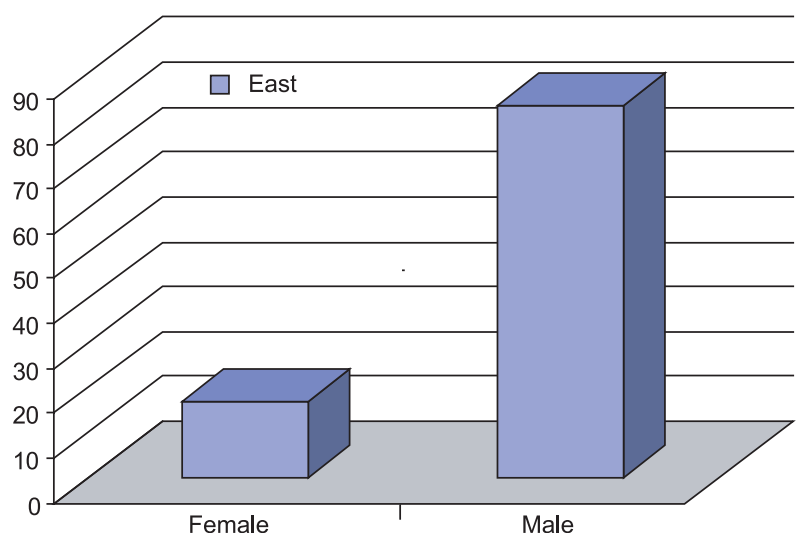

Fig.-2: Sex distribution of referral patients for MPI 
3). The early age group are mostly of female and they have been suffering from DCM instead of ICM. This is confirmed in MPI by normal perfusion pattern in rest and stress images, but there is dilated chamber and reduced EF\% below 34. On the basis of this result all the patient were advised for extensive medical management instead of CAG.

4). The patients between age group 35- 5 years are 22 male and 5 are female. In female patients all have significant reversibility in MPI and advice for revascularization. Among male patients 2 cases showed severe fixed perfusion defect and significant irreversible perfusion defect with severely reduced EF\% and were advised for extensive medical management instead of CAG. The remaining 23 cases in male patient showed variable degree of fixed perfusion defects with significant reversibility and were advice for revascularization.

5). Among the 48 patients of $45-60$ years, there are 28 male and 20 female. Total 5 patients of which 4 male and I female was diagnosed as ICM was confirmed by cardiac scan, which showed

dilated chambers, reduced EF and gross perfusion defect with some reversibility and scattered islands of perfusion defect. One of these ICM patients was advised for extensive medical management due to massive dilatation of $\mathrm{LV}$ and large irreversible and fixed perfusion defect. The remaining $4 \mathrm{ICM}$ patients were acceptable candidate for revascularization. Subsequently after revascularization (PCI or CABG) all of them showed improvement of the $\mathrm{EF} \%$ in echo study on discharge and symptomatic benefit.

6). In the 17 patients of above 60 years age group, showed the cardiac scan result as 2 DCM, 4 ICM, 11 IHD of MI or angina on effort.

7). Pattem of heart disease in referral patients for MPI: DCM: 6(6\%).

ICM: 13(13\%).

Angina on effort: 20(20\%).

Anterior MI 13(13\%).

Inferior MI: 13(13\%).

Anterior and Inferior MI: 35(35\%).

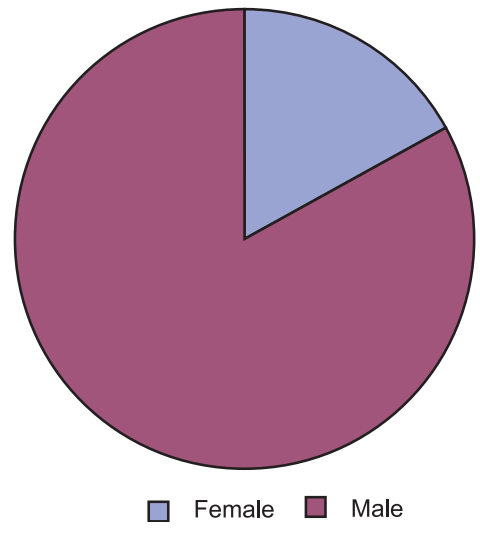

Fig.-3: Distribution of diseases in MPI

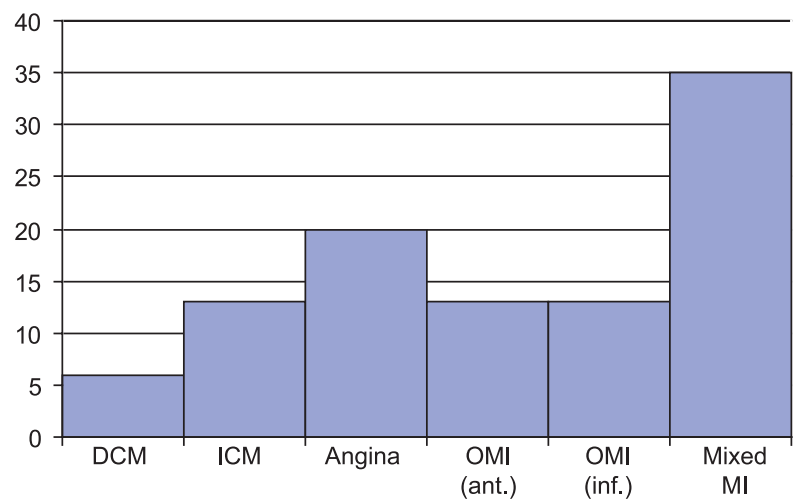

Fig.-4: Distribution of diseases in MPI

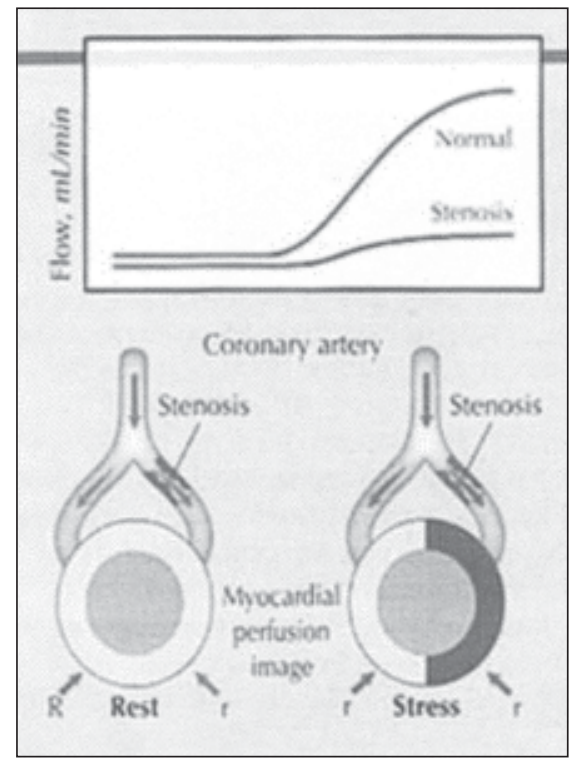

Pathophysiology of underlying radionuclide myocardial perfusion image. Coronary blood flow in each of the coronary artery branches at rest and during stress is shown in the graph.

Fig.-5: Atlas of nuclear cardiology 


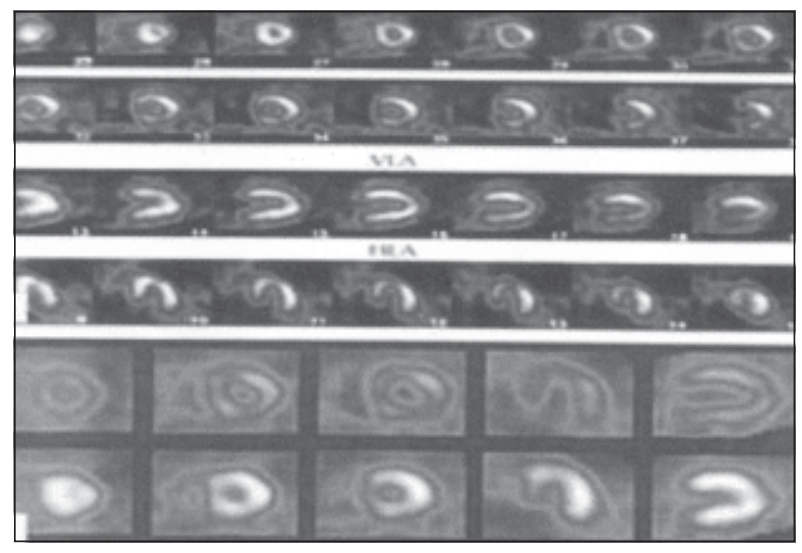

Fig.-6: Normal gated SPECT image.

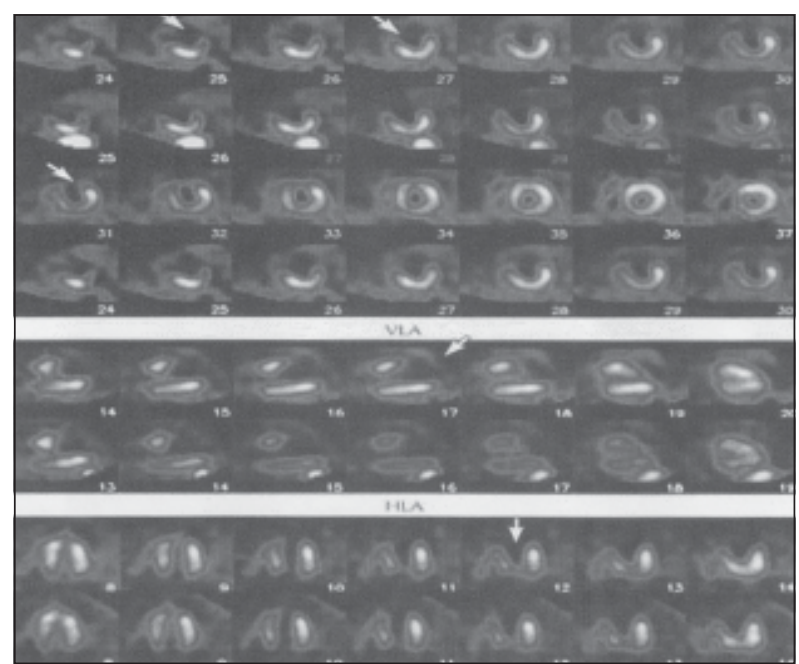

Fig.-7: Anterior \& apical fixed (irreversible) MPI result.

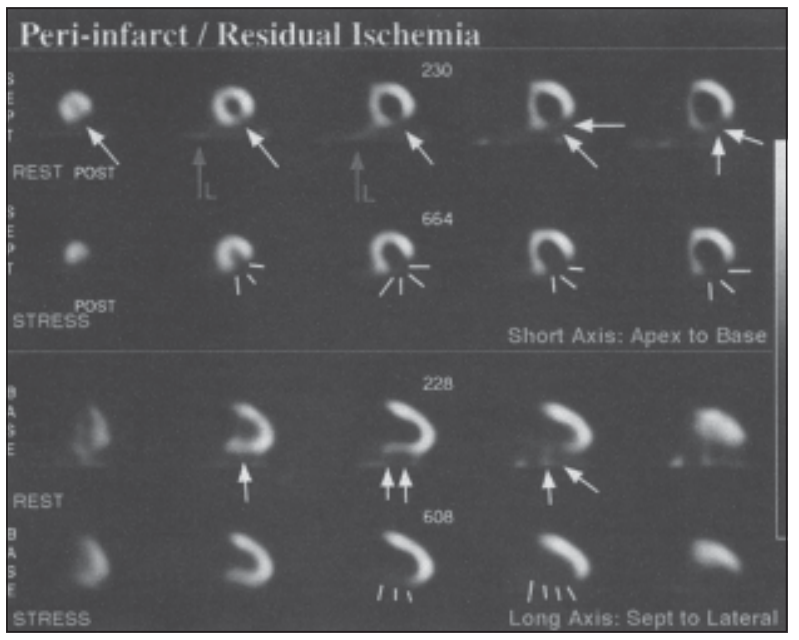

Fig.-8: Post-infarct: residual ischaemia

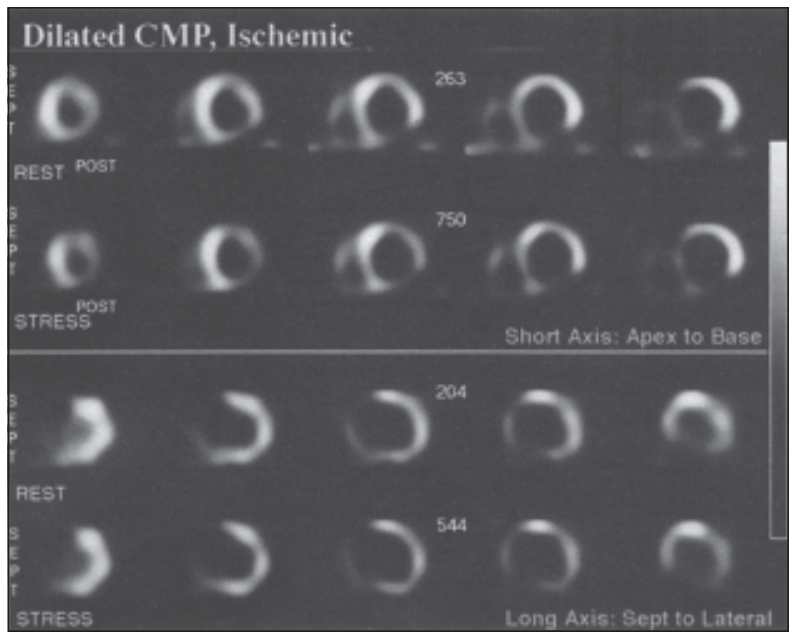

Fig.-9: Dilated CMP, Ischaemic.

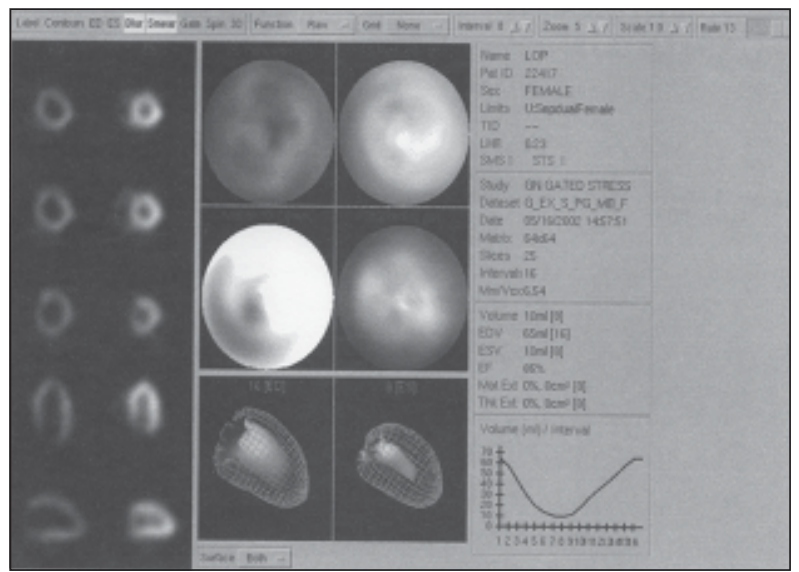

Fig.-10: Cardiac changes in systole and distal.

\section{Discussion:}

The principal applications of gated MPI are related to its superior diagnostic and prognostic performance. Gated MPI I used as a diagnostic tool in the evaluation of patients with suspected CAD.

Diagnostic dilemma of DCM and ICM can be solved by MPI followed by specific treatment of either revascularization or medical treatment.

In case of severe ICM with gross LV dysfunction and massive dilated cardiac chambers showed in echo, some times it is a great decisive dilemma about CAG /or revascularization. Only MPI can solve the problem by displaying reversibility of viable myocardium or stunted myocardium or hibernating myocardium.

In case of multivessels coronary artery disease in $\mathrm{CAG}$, in a diagnose case of OMI(Anterior + Inferior) in $\mathrm{ECG}$, having gross LV dysfunction $(\mathrm{EF} \%<30)$ 
in echo, some times it is a great decisive dilemma about CAG /or revascularization. Only MPI can solve the problem by displaying reversibility of viable myocardium or stunted myocardium or hibernating myocardium.

\section{Conclusion:}

A study of diagnostic and therapeutic value of cardiac scan has been made on series of 100 patients in whom the diagnosis has been confirmed by ECG, X-ray, Echocardiophy, and CAG, also at surgery.

Analysis of perfusion status, decision of subsequent treatment either by medicine or CABG, conform the usually described pattern in western literature.

The commonest findings observed in this present analysis were the early age group patients mostly of female having DCM, but the later age group of patients are of both male and female having Angina Pectoris, OMI and ICM.

The referral patients by cardiologists or cardiac surgeons are mostly limited to the pre therapeutic evaluation rather than diagnostic indication.

The most common indication is the evaluation of myocardial viability and aim of subsequent treatment.

\section{More to be done by MPI in Bangladesh:}

Myocardial perfusion imaging also gaining a role in emergent setting as a tool to diagnose AMI even when ECG and cardiac enzymes are normal or inconclusive.

\section{Conflict of Interest - None.}

\section{References:}

1. Ragosta M,Beller GA: The noninvasive assessment of myocardial viability. Clin cardiol 1993, 16: 531-538.

2. Berman DS, Hayes SW, Germano G: In cardiac spect imaging. Edited by De Peuy EG Garcia EV, Berman DS. Philadelphia: Lippincot Williams and Wilkings; 2000: 179-210.

3. Heo J, Kegel J, Iskandrian AS, et al: Comparison of same-day protocol using technetium 99m Sestamibi myocardial imaging. J Nucl Med 1992, 33: 186-191.

4. Taillefer R, Gagnon A, Laflame L, et al.: Same day injection of Tc. $99 \mathrm{~m}$ methoxy isobutyl isonitril (Hexamibi) for myocardial toographic imaging: comparison between rest-stress and stress-rest injection sequences. Eur J Nucl Med 1989, 15: 113-117.

5. Weiner DA, MC Cabe CH, Ryan TJ: Prognostic assessment of patients with coronary artery disease by exercise testing. Am heart $J$ 1983, 105: 749-755.
6. Mc Neer JF, Margolis JR, Lee KL, et al: The role of exercise testing in the evaluation of patients for ischemic heart disease. Circulation 1978, 57: 64-70.

7. Gould LK. Westcott R, Albro P, et al: Noninvasive assessment of coronary artery stenosis by myocardial imaging during pharmacologic coronary vasodilatation. 11. Clinical methodology and feasibility. Am J Cardiol 1978, 41: 279.

8. Geleijnse ML, Elhendy A, Fioretty PM, Roelandt JR: Dobutamine stress myocardial perfusion imaging. $J$ Am Cardiol 2000, 36: 2017-2027.

9. Cerqueira MD, Weissman NJ, Dilsizian V, et al: Standardized myocardial segmentation and nomenclature for tomographic imaging of the heart: statements for health care professionals from the cardiac magnify committee of the council on clinical cardiology of the American Heart Association. J Nucl Cardiol 2002, 9: 240-245.

10. Van Train, Garcia EV, Cooke DC, Arreda JS: Quantitative analysis of SPECT myocardial perfusion. In: Cardiac SPECT imaging. De Puey EG, Garcia EV, Berman DS (editors). Philadelphia: Lippincot Williams \& Wilkings; 2000: 41-64.

11. Berman DS, Hachamovitch R, Kiat H, et al: Incremental value of prognostic testing patients with known or suspected ischemic heart disease: A basis for optimal utilization of exercise technetium $99 \mathrm{~m}$ Sestamibi myocardial perfusion single photon emission computed tomography. Am J Cardiol 1995, 26: 639-647.

12. Germano G, Kiat H, KavanaghPB, et al: Automatic quantification of ejection fraction from gated myocardial perfusion SPECT. J Nucl Med 1994, 35: 1185-1192.

13. Farber T, Cooke C, Folks R, et al: Left ventricular function and perfusion from gated SPECT perfusion images: an integrated method. J Nucl Med 1999, 40: 650-659.

14. Vaduganathan P, He Z, Vick, et al: Evaluation of left ventricular wall motion, volumes and ejection fraction by gated myocardial tomography with technitium $99 \mathrm{~m}$ labeled tetrofosmin: a comparison with cine magnetic resonance imaging. J Nucl Cardiol 1999, 6: 3-10.

15. Lewin HC, Berman DS, Hayes HW et al: Clinical reproducibility of post-stress gated myocardial perfusion SPECT imaging. J Nucl Med 2000, 41: 160P. 16. Sharir T, Bacher-Steir C, Dhar S, et al: Identification of severe and extensive coronary artery disease by post exercise regional wall motion abnormalities in technitium $99 \mathrm{~m}$ Sestamibi gated single photon emission computed tomography. Am J Cardiol 2000, 86: 1171-1175.

17. Cuocolo A, Pace L, Ricciardelli B, et al: Identification of viable myocardium in patients with chronic coronary artery disease comparison of TI 201 scintigraphy with reinjection and technitium $99 \mathrm{~m}$ methoxyisobutyl isonitrile. J Nucl Med 1992, 33: 505-511. 
18. Shirani J, Alaeddini J, Pick R, and Dilsizian V: Variations in collagen content of synergic left ventricular segments in explanted hearts of man with ischemic cardiomyopathy. Am J Cardiol 2002, 89: 865-869.

19. Bennett SK, Smith MF, Gottlieb SS, et al.: Effect of metoprolol on absolute myocardial blood flow in patients with heart failure secondary to ischemic or non-ischemic cardiomyopathy. Am J Cardiol 2002, 89: 1431-1434.

20. Allman KC, Shaw U, Hachamovitch R, Udelson JE: Myocardial viability testing and impact of revascularization on prognosis in patients with coronary artery disease and left ventricular dysfunction: a metaanalysis. J Am Coll Cardiol 2002, 39: 1151-1158.

21. Samady H, Wackers FJ, and Joska TM, et al.: Pharmacologic stress perfusion imaging with adenosine: role of simultaneous low-level treadmill exercise. $\mathrm{J} \mathrm{Am}$ Coll Cardiol 2002, 9: 188-196.

22. Gibbons RJ, Miller TD, Chritian TF: Infarct size measured by single photon emission computed tomographic imaging with $(99 \mathrm{~m})$ Tc-Sestamibi: A measure of the efficacy of therapy in acute myocardial infarction. Circulation 2000, 101(1): 101-108. 